\title{
Release Dependence to a Paired Stimulus at a Synaptic Release Site with a Small Variable Pool of Immediately Releasable Vesicles
}

\author{
Eric Hanse and Bengt Gustafsson \\ Institute of Physiology and Pharmacology, Göteborg University, SE-405 30 Göteborg, Sweden
}

\begin{abstract}
Monte Carlo simulations were performed on a release model based on experimental data from single glutamatergic synapses containing a single release site in the hippocampal CA1 region of the neonatal rat. These simulations explored what can be learned about the release process by examining how the release probability in response to the second stimulus $\left(P_{2}\right)$ of a paired stimulus to a synapse depends on the release in response to the first stimulus. Comparisons between experimental data from a number of individual synapses and the simulated
\end{abstract}

data support the notion that the immediately releasable vesicle pool is small (approximately one) and shows substantial intertrial variation. The simulations also show that the release dependence of $P_{2}$ is not necessarily an indicator of either intertrial variation in $\mathrm{Ca}^{2+}$ influx, feedback effects of released transmitter, or activation failure of the axon.

Key words: paired-pulse; release probability; hippocampus; CA1; synaptic plasticity; development
The physiology of the release process of a synapse is still understood primarily in terms of number of release sites and release probabilities at these sites. However, linking the molecular biology of the release machinery to the physiology of release will require an understanding of what determines release probability at a single release site. In a recent examination of neonatal rat hippocampal synapses containing a single release site (Hanse and Gustafsson, 2001b), release was described in terms of intrarelease site parameters, such as vesicle release probability, number of immediately releasable vesicles, and vesicle recruitment (Hanse and Gustafsson, 2001a,c,d). This description indicates that a small number (which fluctuated from trial to trial) of immediately releasable vesicles (usually fewer than three) independently, and with the same probability, contribute to the release of at most one vesicle at a time. Such characteristics should have consequences for the serial dependency of release: for example, in what manner release in response to the first stimulus of a paired stimulus affects the release probability to the second stimulus $\left(P_{2}\right)$. Such a test has been used to detect whether release failure to the first stimulus is present because of the stochastic nature of the release process itself or because of failure to activate the axon/synaptic bouton (Stevens and Wang, 1995; Isaac et al., 1996; Hanse and Gustafsson, 2001b). That is, if $P_{2}$ depends on whether or not there is release failure in response to the first stimulus, then this release failure should be attributable to activation failure.

Although the above may be true, for example, if the number of immediately releasable vesicles at a single site is large (Dobrunz and Stevens, 1997), it would not be expected to occur if that number is small. This is because a small pool of such vesicles would be greatly depleted by a release to the first stimulus causing

\footnotetext{
Received Nov. 29, 2001; revised March 11, 2002; accepted March 18, 2002.
}

This project was supported by the Swedish Medical Research Council (Project Numbers 12600 and 05180), the Swedish Society of Medicine, and Harald Jeanson's Foundation. We thank F. Asztely, L. Groc, and P. Wasling for critically reading this manuscript.

Correspondence should be addressed to Eric Hanse, Institute of Physiology and Pharmacology, Göteborg University, Box 432, SE-405 30 Göteborg, Sweden. E-mail: eric.hanse@physiol.gu.se.

Copyright (C) 2002 Society for Neuroscience $0270-6474 / 02 / 224381-07 \$ 15.00 / 0$ release-dependent effects on $P_{2}$ even in the absence of activation failure. Release dependence may also arise if presynaptic calcium influx varies between stimulus trials, thereby linking release to the first stimulus to facilitation of release to the second one (Chen et al., 1996; Dobrunz et al., 1997). Moreover, multivesicular release from a single site or heterogeneity in release probability among the vesicles within a site might also have consequences for the release dependence of $P_{2}$.

Considering the possibilities for release dependence of $P_{2}$ in the absence of activation failure, it may seem surprising that no such dependence was observed (Stevens and Wang, 1995; Isaac et al., 1996; Hjelmstad et al., 1997; Hanse and Gustafsson, 2001b). This raises the question of in what manner and to what extent the above-mentioned factors actually affect the release dependence; in other words, to what extent an examination of the release dependence of $P_{2}$ may serve to discriminate between different models of presynaptic release. To answer such questions, we performed Monte Carlo simulations of release from a model single release site. We also compared these simulated results with those from experimental data on release from single glutamatergic hippocampal synapses of neonatal rats.

\section{MATERIALS AND METHODS}

The experimental data used in the present study were obtained in a manner that has been described previously in detail (Hanse and Gustafsson, 2001b). In brief, whole-cell patch-clamp recordings were performed from visually identified CA1 pyramidal cells in hippocampal slices prepared from 1- to 7-d-old Wistar rats. Afferents in the stratum radiatum were activated (10 impulses; $50 \mathrm{~Hz}$ ) using minimal extracellular stimulation (Konnerth et al., 1990; Raastad et al., 1992; Allen and Stevens, 1994). Several findings suggested that this minimal stimulation consistently resulted in the activation of a single axon contributing a single synapse to the cell that was recorded from, with this synapse containing a single release site (Hanse and Gustafsson, 2001b). To what extent such a single site releases at most one or several vesicles at a time is a matter of debate (Auger and Marty, 2000). Two lines of experimental findings support the single-vesicle constraint at glutamatergic synapses: first, that release is accompanied by a refractoriness of release (Stevens and Wang, 1995; Dobrunz et al., 1997; Hjelmstad et al., 1997), and second, that EPSC size, excluding failures, is independent of release probability (Stevens and Wang, 1995; Dobrunz and Stevens, 1997; Hjelmstad et al., 1997; Hanse and Gustafsson, 2001b). This latter evidence presupposes a 
low degree of postsynaptic receptor occupancy, allowing for the detection of more than one simultaneously released vesicle. Experimental support for such a low degree of occupancy has been published recently (Liu et al., 1999; McAllister and Stevens, 2000; Barbour, 2001; Hanse and Gustafsson, 2001b).

Release model. In our standard release model, release probability $\left(P_{\mathrm{r}}\right)$ at a single release site is determined by the number of release-ready, or primed, vesicles $\left(n_{\text {pool }}\right)$ and the probability of release of each one of these vesicles $\left(P_{\text {ves }}\right)$. These primed vesicles operate independently and with the same $P_{\text {ves }}$ value to produce release of a single vesicle:

$$
P_{\mathrm{r}}=1-\left(1-P_{\text {ves }}\right)^{n_{\text {pool }}} \text {. }
$$

Because this equation describes release probability as 1 minus the probability that no release occurs, it allows for multivesicular release. However, whether the release is univesicular or multivesicular is naturally of consequence for the rate of depletion of the pool. On the basis of our finding that EPSC size, excluding failures, in these synapses is independent of release probability in a given synapse (Hanse and Gustafsson, 2001b) and the notion of a low degree of postsynaptic receptor occupancy (see above), our standard release model will contain the singlevesicle constraint. However, in our simulations, we will also explore the consequences of relaxing this constraint, allowing for multivesicular release.

An experimental analysis of release from single synapses allowed us to estimate $P_{\text {ves }}$ and $n_{\text {pool }}$ for a number of synapses (Hanse and Gustafsson, 2001c). $P_{\text {ves }}$ for release given by the first stimulus $\left(P_{\text {ves } 1}\right)$ was found to vary among the synapses from $<0.1$ to almost 1.0. Conversely, $P_{\text {ves }}$ for release given by the second stimulus $\left(P_{\text {ves } 2}\right)$ varied less, $\sim 0.2-0.5$ (Hanse and Gustafsson, 2001a). The average number of vesicles immediately available for release at the initiation of the stimulus train, here referred to as the preprimed pool, was found to vary among the synapses from $\sim 0.5$ to 2.0 . Additional analysis suggested that this pool varied between trials in a binomial manner (Hanse and Gustafsson, 2001d).

A key feature in our release model is that the number of immediately releasable vesicles is determined by docked vesicles in equilibrium between a primed and a nonprimed state (Fig. 1 $A$ ) (Matveev and Wang, 2000; Hanse and Gustafsson, 2001d). Thus, the number of vesicles in the preprimed pool will vary between trials in a binomial manner (Fig. $1 B$ ). To obtain preprimed pool sizes in the model in agreement with the experimentally estimated ones, the number of docking sites was varied from 2 to 6 , and the probability of the primed state was set to 0.3 . On the basis of the experimentally estimated values, $P_{\text {ves } 1}$ was allowed to vary between 0.1 and 0.9 in the simulations, and $P_{\text {ves2 }}$ was set to a fixed value of 0.4 (Hanse and Gustafsson, 2001a). The Monte Carlo simulations were performed using custom software written in Igor Pro (Wavemetrics, Lake Oswego, OR). Simulations were performed such that before each simulated paired stimulus (trial), the number of preprimed vesicles was determined by comparing a random number (evenly distributed between 0 and 1) with the preset value of priming probability for each docking site. If the random number was smaller than that value, the vesicle was considered to be primed. To determine whether release occurred in the response to the first stimulus, a random number (as above) was compared with the preset $P_{\text {ves } 1}$ for primed vesicles. If the random number was smaller than that value, release occurred, and the preprimed pool was reduced by 1 (under singlevesicle constraints). Conversely, if it was larger than that value, the next (if any) preprimed vesicle was tested. When a release occurred in response to the first stimulus, or if all preprimed vesicles were tested without any one vesicle being released, the occurrence of release in response to the second stimulus was tested by comparing a random number with $P_{\text {ves } 2}$ for each remaining preprimed vesicle. Unless otherwise stated, the number of trials per measurement was 10,000 .

These calculations assume that only preprimed vesicles are released in response to the first two stimuli (i.e., that there is no priming of docked vesicles during a $20 \mathrm{msec}$ interstimulus interval). This assumption is compatible with our previous analysis of experimental data, indicating that most of the release during the first four to five stimuli in a $50 \mathrm{~Hz}$ stimulus train comes from the preprimed pool (Hanse and Gustafsson, 2001c).

\section{RESULTS}

Experiments on single glutamatergic CA1 hippocampal synapses in the neonatal rat have indicated that the immediately releasable vesicle pool (preprimed pool, see Materials and Methods) for a release site is small, on average approximately one (Hanse and

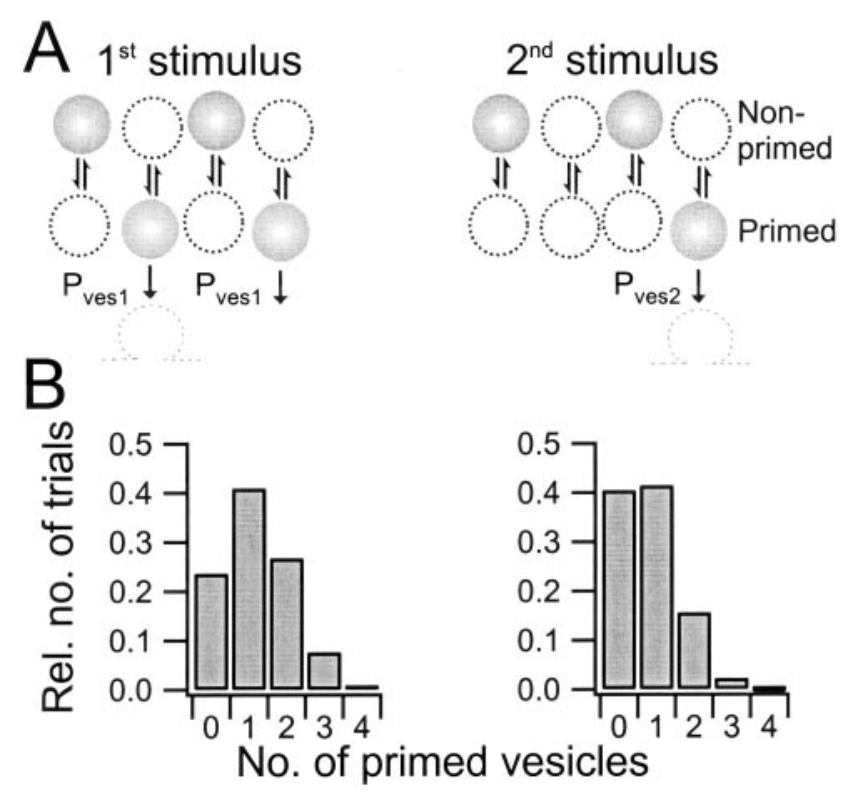

Figure 1. Model for a dynamic pool of primed vesicles at a release site. $A$, Schematic of a model release site at the first (left) and at the second (right) stimuli during a paired-pulse protocol. Within the release site, there are docking sites (four in the illustrated example) at which vesicles are in equilibrium between a primed (immediately releasable) and a nonprimed state. When an action potential arrives, at most one of the primed vesicles can be released. The probability of releasing a primed vesicle is $P_{\text {ves }}, P_{\text {ves } 1}$ at the first stimulus, and $P_{\text {ves } 2}$ at the second stimulus. The $P_{\mathrm{r}}$ of the release site is given by Equation 1 (see Materials and Methods). It is assumed that the recruitment of nonprimed vesicles to a primed state is negligible between the two stimuli $(20 \mathrm{msec})$ (Hanse and Gustafsson, 2001a). B, Distribution of the number of primed vesicles among trials before the first stimulus (preprimed vesicles, left) and before the second stimulus (right). The distribution is calculated from four docking sites and a probability of the primed state of 0.3 .

Gustafsson, 2001c). If so, one may expect that the $P_{\mathrm{r}}$ to a second stimulus $\left(P_{2}\right)$ will depend on release to the first stimulus in a stimulus pair even when there are no activation failures, because of vesicle depletion. We therefore first simulated release using a preprimed pool of 1.2 (on average), letting vesicle release probability during the first $\left(P_{\mathrm{ves} 1}\right)$ and second $\left(P_{\mathrm{ves} 2}\right)$ stimuli have the same value $(0.4)$. The $P_{2}$ values when release occurred in response to the first stimulus $\left(P_{2 \text { rel }}\right)$ and when it did not $\left(P_{2 \text { fail }}\right)$ were subsequently computed. Such a simulation did not demonstrate any release dependence of $P_{2}$ on release to the first stimulus, with the ratio $P_{2 \text { rel }} / P_{2 \text { fail }}$ being close to 1 (1.03). This lack of the "expected" depletion effect may be explained by the fact that the simulated preprimed pool, in agreement with our interpretation of the experimental data, is not constant trial for trial but has a binomially distributed trial-to-trial variation in size (Fig. 1B). Trials with a larger preprimed pool size are more likely to release in response to the first stimulus than trials with smaller (or zero) pool size. The preprimed pool remaining after both release and failure to the first stimulus may then be much the same, as also indicated by Figure $2 A$ (insets).

To examine the reliability of an experimentally estimated $P_{2 \text { rel }} /$ $P_{2 \text { fail }}$ ratio, the above simulation was repeated 100 times (100 runs), using a smaller number of simulated trials than used above $(10,000)$. The ratio estimated at each run when only 100 trials were used varied considerably, from $\sim 0.5$ to 1.5 (Fig. $2 A$ ). Figure $2 B$ shows the coefficient of variation $(\mathrm{CV})$ plotted against the number of trials per run, indicating a $\mathrm{CV}$ value of $\sim 0.4$ ( filled 


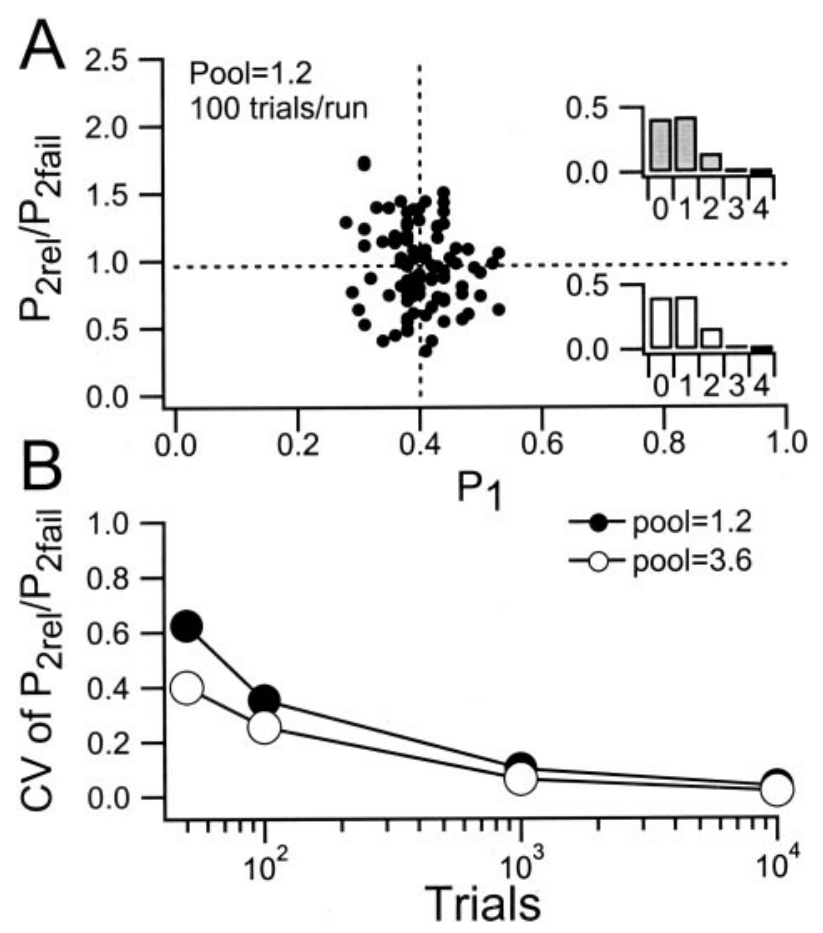

Figure 2. Lack of release dependence during paired-pulse activation. $A$, $P_{\mathrm{r}}$ in response to the second stimulus $\left(P_{2}\right)$ when release occurred in response to the first stimulus $\left(P_{2 \text { rel }}\right)$ is divided by that obtained when there was a release failure to the first stimulus $\left(P_{2 \text { fail }}\right)$. This $P_{2 \text { rel }} / P_{2 \text { fail }}$ ratio is plotted against $P_{\mathrm{r}}$ in response to the first stimulus $\left(P_{1}\right)$. In the modeled example, the preprimed pool was 1.2 , and both $P_{\text {ves } 1}$ and $P_{\text {ves } 2}$ were 0.4 . Ratio values obtained from 100 runs, each consisting of 100 trials, are shown. The $P_{2 \text { rel }} / P_{2 \text { fail }}$ ratio averaged $0.96 \pm 0.35(\mathrm{SD})$, and $P_{1}$ averaged $0.40 \pm 0.05$. These average values are indicated by the dotted lines. Insets show the distribution of primed vesicles (compare Fig. $1 B$ ) before the second stimulus when the first stimulus resulted in a release (top histogram, filled bars) and when the first stimulus resulted in a failure (bottom histogram, open bars). $B$, Variation in $P_{2 \text { rel }} / P_{2 \text { fail }}$ ratio as a function of the number of trials and of the size of the preprimed pool. The number of trials per run was varied between 50 and 10,000 , and 100 runs were made for each trial length. Filled circles represent a pool of 1.2 and open circles represent a pool of 3.6.

circles). Even with 1000 trials per run, a notable $\mathrm{CV}$ is found $(\sim 0.10)$, and several thousand trials are needed to bring the $\mathrm{CV}$ to a value of $<0.05$. An increase of the preprimed pool increased the reliability, but not much. For example, when an average pool three times larger (3.6; with a threefold reduction of $\left.P_{\text {ves }}\right)$ was used, 100 and 1000 trials gave CVs of $\sim 0.25$ and 0.05 , respectively (Fig. 2B, open circles).

\section{Influence of variation in $\boldsymbol{P}_{\text {ves } 1}$ and preprimed pool size on the release dependence of $\boldsymbol{P}_{2}$}

To examine to what extent the above result was a consequence of the particular choice of $P_{\mathrm{ves} 1}$ and preprimed pool values, the simulations were performed using the range of these values obtained from experimental data on the neonatal CA1 synapses. The $P_{2 \text { rel }} / P_{2 \text { fail }}$ ratio was thus plotted against $P_{1}$ values obtained by varying $P_{\text {ves } 1}$ from 0.1 to 0.9 and the average preprimed pool from 0.6 to 1.8 . This ratio was found to vary with $P_{1}$ and to be close to 1.0 only at intermediate values of $P_{1}(\sim 0.4)$ (Fig. $\left.3 A\right)$. At lower values of $P_{1}$, the $P_{2 \text { rel }} / P_{2 \text { fail }}$ ratio is $<1.0$, indicating that $P_{2}$ is less when release occurs in response to the first stimulus than when it does not. Conversely, when $P_{1}$ is larger, the ratio becomes substantially more than 1.0 , indicating the opposite. It would thus
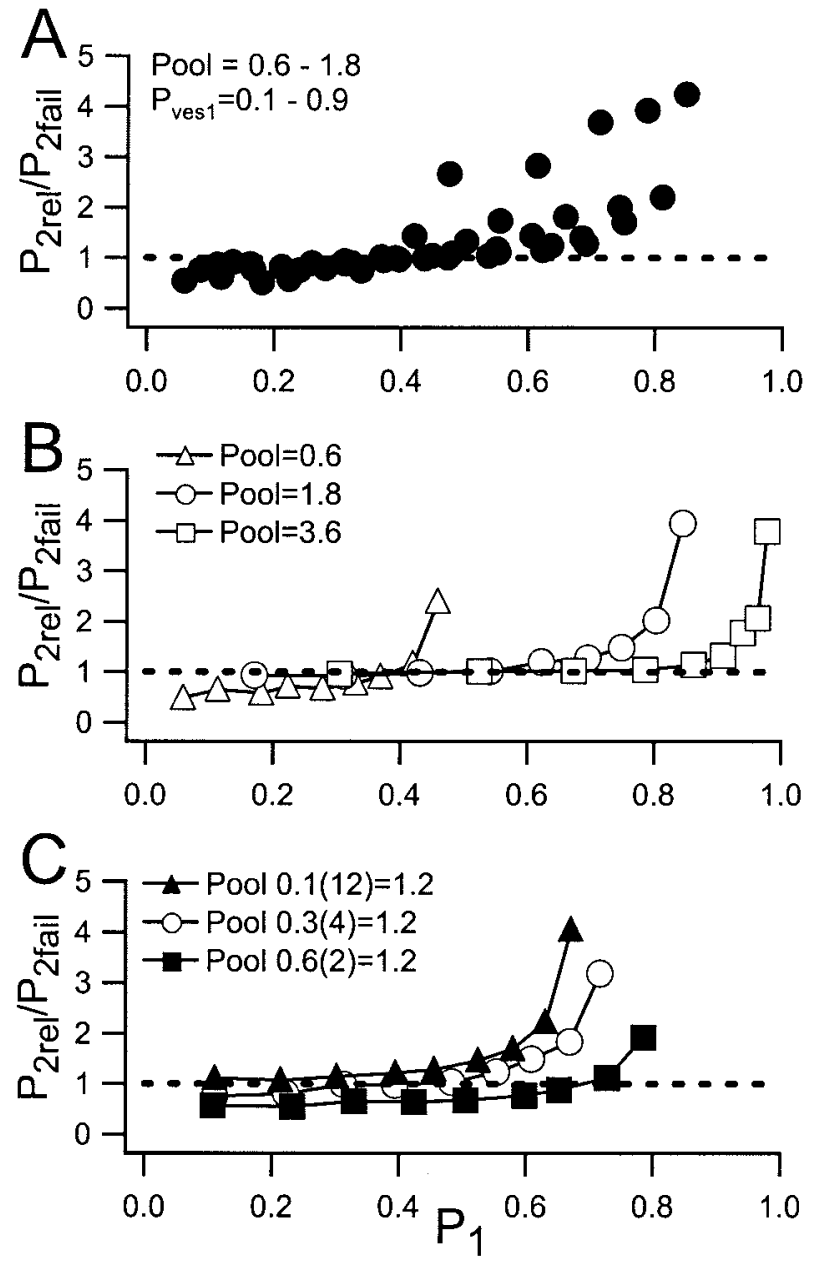

Figure 3. Influence of $P_{\mathrm{r}}$ on the release dependence during paired-pulse activation. $A$, The $P_{2 \text { rel }} / P_{2 \text { fail }}$ ratio is computed over a range of $P_{1}$ values obtained by varying $P_{\text {ves } 1}$ between 0.1 and 0.9 (in steps of 0.1 ) and the average preprimed pool between 0.6 and 1.8 (in steps of 0.3 ). $P_{\text {ves2 } 2}$ was kept constant at 0.4 . The dashed line indicates release independence (i.e., a $P_{2 \text { rel }} / P_{2 \text { fail }}$ ratio equal to 1$)$. Each measurement was obtained from 10,000 trials. $B$, The $P_{2 \text { rel }} / P_{2 \text { fail }}$ ratio is determined by $P_{\text {ves } 1}$ and the size of the preprimed pool. The $P_{2 \text { rel }} / P_{2 \text { fail }}$ ratio is plotted against $P_{1}$ obtained using three different preprimed pool sizes: 0.6 (open triangles), 1.8 (open circles), and 3.6 (open squares). For each pool size, $P_{\mathrm{ves} 1}$ was varied from 0.1 to 0.9 in steps of 0.1 , and the $P_{2 \text { rel }} / P_{2 \text { fail }}$ ratios obtained for the various $P_{\mathrm{ves} 1}$ values using a given pool size are joined together. The dashed line indicates $P_{2 \text { rel }} / P_{2 \text { fail }}=1$. $C$, The $P_{2 \text { rel }} / P_{2 \text { fail }}$ ratio depends on the number of docked vesicles and their probability of occupancy of the primed state. The $P_{\text {2rel }} / P_{\text {2fail }}$ ratio is plotted against $P_{1}$, varying $P_{\text {ves } 1}$ from 0.1 to 0.9 in steps of 0.1 . This is done for three different preprimed pool configurations, all producing an average preprimed pool of 1.2 vesicles. These configurations are 12 (filled triangles), 4 (open circles), and 2 (filled squares) docked vesicles combined with priming probabilities of $0.1,0.3$, and 0.6 , respectively. The dashed line indicates $P_{2 \text { rel }} / P_{2 \text { fail }}=1$.

appear that when $P_{1}$ is large, a smaller number of vesicles are available for release to the second stimulus when there is no release in response to the first stimulus than when there is a release. This perhaps paradoxical result can be explained by the trial-to-trial variation in the preprimed pool and by the fact that a larger $P_{1}$ is associated with a larger $P_{\text {ves } 1}$, because when $P_{\text {ves } 1}$ is large, it is very probable that trials exhibiting release failure in response to the first stimulus are associated with a small number of (or zero) preprimed vesicles and subsequently also with release failure in response to the second stimulus. Conversely, a small $P_{1}$ 
is associated with a small $P_{\mathrm{ves} 1}$. Release failure in response to the first stimulus then depends more on the small $P_{\text {ves1 }}$ than on the size of the preprimed pool, and vesicle depletion will more critically affect $P_{2}$. To substantiate this reasoning, the results of the simulation in Figure $3 A$ are replotted in Figure $3 B$, using only those values that were obtained with the smallest (0.6) and largest (1.8) preprimed pool, together with simulation results using an even larger pool (3.6). Figure $3 B$ shows that for any given pool size, the $P_{2 \text { rel }} / P_{2 \text { fail }}$ ratio goes from $<1.0$ to $>1.0$ when $P_{\text {ves } 1}$ goes from a small to a large value. Moreover, when the pool becomes larger, the ratio becomes closer to 1.0 for any value of $P_{1}$. In these simulations, $P_{\text {ves2 }}$ was set to a fixed value of 0.4 (Hanse and Gustafsson, 2001a). Variations of this parameter over a wide range (0.1-0.9) had only marginal effects on the $P_{2 \text { rel }} / P_{2 \text { fail }}$ ratio (data not shown). This is because in the range of pool sizes used, pool size and $P_{\text {ves2 }}$ will act almost linearly to produce $P_{2}$.

In the above simulations, a preprimed pool of 1.2 was set by four docked vesicles in equilibrium between a primed and a nonprimed state, with a probability of the primed state of 0.3 . A smaller docked pool with a larger priming probability and vice versa could produce the same averaged value. Figure $3 C$ shows simulations using 2, 4, and 12 docking sites to obtain an average preprimed pool of 1.2. The use of both the small (filled squares) and the large ( filled triangles) number of sites created curves with larger deviation from a unity ratio (i.e., a larger degree of release dependence of $P_{2}$ ).

\section{Influence of $\boldsymbol{P}_{\text {ves }}$ heterogeneity on the release dependence of $\boldsymbol{P}_{2}$}

The above-described simulations were all performed with the assumption that in any given run, $P_{\text {ves } 1}$ is the same between trials and $P_{\text {ves } 1}$ at each trial is equal for all the preprimed vesicles within a release site. For a synapse, the $\mathrm{Ca}^{2+}$ influx may vary between trials and thus cause a variation in $P_{\text {ves1 }}$ as well as (via residual $\mathrm{Ca}^{2+}$ ) in $P_{\text {ves2 }}$ (Chen et al., 1996; Dobrunz et al., 1997). $P_{\text {ves2 }}$ would then be expected to be larger when $P_{\text {ves } 1}$ is larger, and a release dependence of $P_{2}$ would emerge. A variation in $\mathrm{Ca}^{2+}$ influx was simulated by a normal distribution (SD 0.2) around a mean $P_{\text {ves } 1}$ that was $0.2,0.4,0.6$, or 0.8 . The $P_{\text {ves } 1}$ value for each trial was thus determined by a stochastically drawn value, as exemplified in Figure $4 A$, inset, for the case of a mean $P_{\text {ves } 1}$ of 0.4 . To account both for the effect of residual $\mathrm{Ca}^{2+}$ and for variation in $\mathrm{Ca}^{2+}$ influx to the second stimulus, the $P_{\text {ves } 2}$ value of 0.4 was adjusted by two stochastically drawn values for each trial. Residual $\mathrm{Ca}^{2+}$ was accounted for by letting $P_{\text {ves } 2}$ be adjusted by the same stochastically drawn value that helped to decide $P_{\text {ves1 }}$ for that trial. The variation in $\mathrm{Ca}^{2+}$ influx was accounted for by also adjusting $P_{\text {ves } 2}$ with a value stochastically drawn from the same

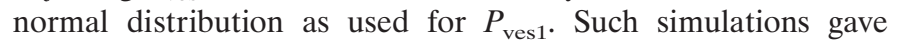
$P_{2 \text { rel }} / P_{2 \text { fail }}$ ratios that were shifted to $30-40 \%$ higher values than those obtained in the absence of this form of heterogeneity, as illustrated in Figure $4 A$. That is, such a variation would produce substantial release dependence.

Vesicles within a release site may differ in their release probability (for example, one of them having a much larger $P_{\text {ves }}$ than the others) (Matveev and Wang, 2000; Sakaba and Neher, 2001). $P_{2}$ would then vary depending on whether or not that specific vesicle was released in response to the first stimulus. Simulations were performed that allowed one of the docking sites to contain a primed vesicle with a high $P_{\text {ves}}$, if occupied. In the first case, this high $P_{\text {ves }}(0.9)$ applied only to $P_{\text {ves } 1}$, and the simulations were performed for three different pool sizes. The $P_{\mathrm{ves} 1}$ for the other
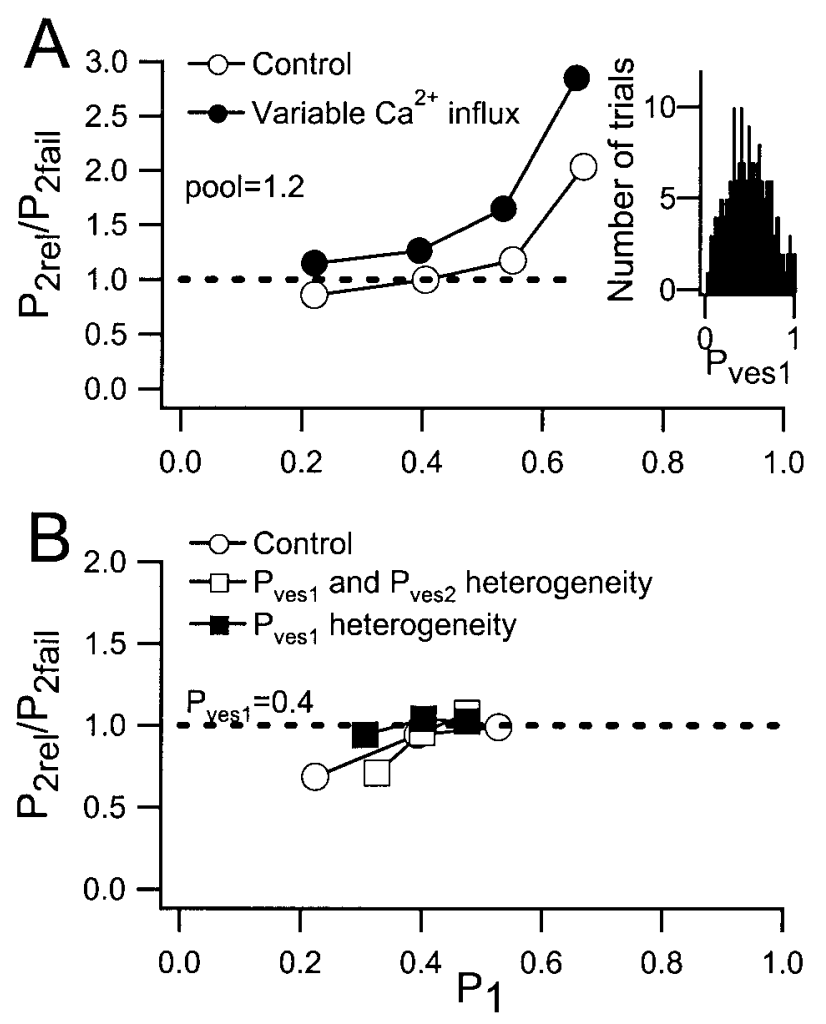

Figure 4. Influence of $P_{\text {ves }}$ heterogeneity on the release dependence during paired-pulse activation. $A$, Effect of a stochastic variation in $P_{\text {ves } 1}$ and $P_{\text {ves } 2}$ on the release dependence of $P_{2}$. The $P_{2 \text { rel }} / P_{2 \text { fail }}$ ratio is plotted against $P_{1}$ for two different situations. Open circles represent the release dependence of $P_{2}$ in a control situation using four different values of $P_{\text {ves } 1}$ $(0.2,0.4,0.6$, and 0.8$)$, and $P_{\text {ves } 2}$ was kept constant at 0.4. Filled circles represent the release dependence of $P_{2}$ when $P_{\mathrm{ves} 1}$ and $P_{\mathrm{ves} 2}$ were allowed to vary stochastically around these values, as described in Results. The dashed line indicates $P_{2 \text { rel }} / P_{2 \text { fail }}=1 . B$, Different docking sites are associated with different $P_{\text {ves }}$ values. Open circles represent a control situation with $P_{\mathrm{ves} 1}$ and $P_{\mathrm{ves} 2}$ values of 0.4 at all the docking sites. Filled squares represent a situation in which one of the two to six docking sites was associated with a $P_{\text {ves1 }}$ of 0.9 , whereas the other docking sites were associated with a $P_{\text {ves } 1}$ of $0.2 . P_{\text {ves } 2}$ was kept constant at 0.4 for all docking sites. Open squares represent a situation in which one of the two to six docking sites was associated with a $P_{\text {ves } 1}$ and $P_{\text {ves } 2}$ of 0.9 , whereas the others were associated with a $P_{\text {ves1 }}$ and $P_{\text {ves2 }}$ of 0.2 . The dashed line indicates $P_{2 \text { rel }} / P_{2 \text { fail }}=1$.

vesicles was 0.2 , and the $P_{\text {ves } 2}$ for all vesicles was 0.4 . Figure $4 B$ shows that this type of heterogeneity (filled squares) produces a relationship between the $P_{2 \text { rel }} / P_{2 \text { fail }}$ ratio and $P_{1}$ that is close to that obtained by a homogeneous $P_{\text {ves } 1}$ of 0.4 (open circles). In the second case, the high- $P_{\text {ves }}$ vesicle also had a $P_{\text {ves } 2}$ of 0.9 , and the $P_{\text {ves } 2}$ for the others was 0.2 . This heterogeneity also produced a relationship between the $P_{2 \text { rel }} / P_{2 \text { fail }}$ ratio and $P_{1}$ (open squares) that was close to the homogeneous case. Thus, $P_{\text {ves }}$ heterogeneity is not easily revealed by this test of release dependence.

\section{Influence of activation failure and of multivesicular release on the release dependence of $\boldsymbol{P}_{2}$}

The test of release dependence was originally thought of as a means to detect activation failure (see the introductory remarks). An estimation of the effect of activation failure requires simplification of assumptions regarding activation failures in response to the first and second stimuli. Because it would seem that the method is based on the notion that activation failures in response to the second stimulus occur independently of those to the first 

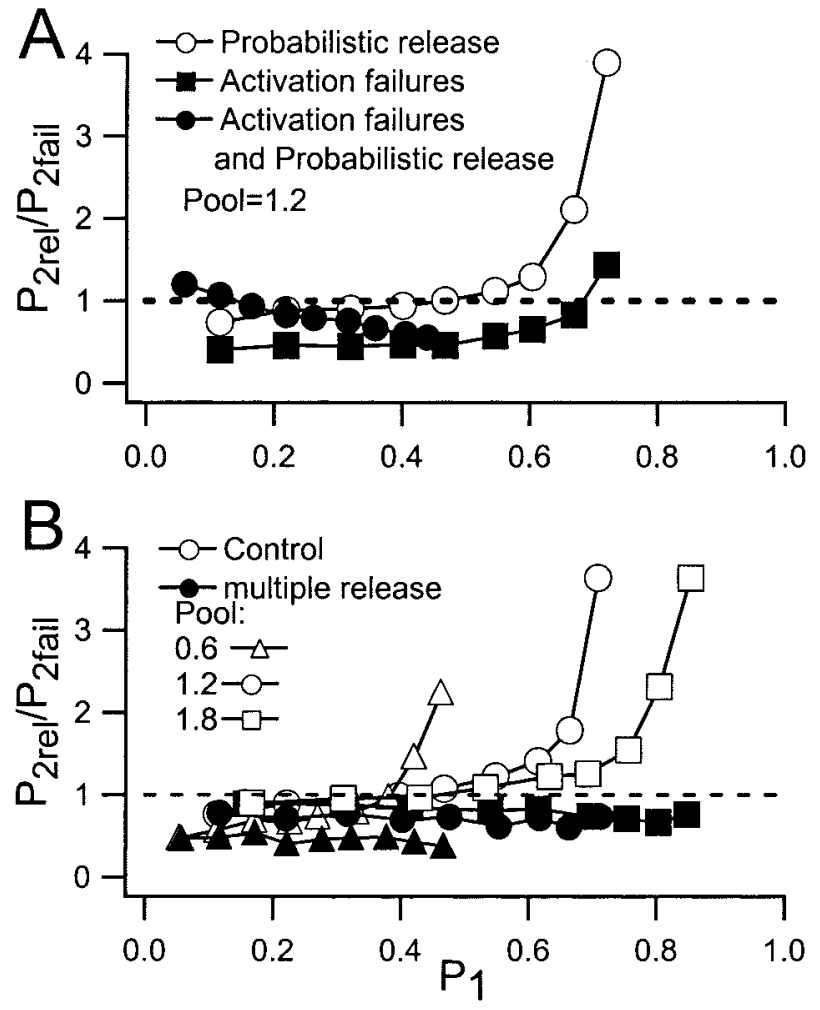

Figure 5. Influence of activation failure and multivesicular release on the release dependence during paired-pulse activation. $A$, The $P_{2 \text { rel }} / P_{2 \text { fail }}$ ratio is plotted against $P_{1}$ for two different situations of activation failure. Open circles represent the release dependence of $P_{2}$ in a control situation with no activation failure (i.e., a "probabilistic release"). $P_{\text {ves1 }}$ is varied from 0.1 to 0.9 , and the preprimed pool and $P_{\text {ves } 2}$ are kept constant at 1.2 and 0.4 , respectively (compare Fig. $3 C$, open circles). Filled circles represent a situation in which $50 \%$ of the trials were associated with an activation failure (no release is allowed) in response to the first stimulus; in other words, activation failures occur on top of a probabilistic release. $P_{\text {ves } 1}, P_{\text {ves2 } 2}$, and pool were the same as in the control situation. Filled squares represent a situation in which $P_{1}$ is determined by activation failures only. In this situation, $P_{\text {ves } 1}$ was (per definition) equal to 1 , and $P_{\text {ves2 }}$ (used only when there was activation and hence release in response to stimulus 1) was equal to 0.4 . The pool size was 1.2 . The dashed line indicates $P_{2 \text { rel }} / P_{2 \text { fail }}=1 . B$, The effect of allowing multiple release of vesicles on the $P_{2 \text { rel }} / P_{2 \text { fail }}$ ratio. Open symbols represent the control situation, in which at most one vesicle per stimulus is released. $P_{\text {ves } 1}$ is varied from 0.1 to 0.9 for three different sizes of the preprimed pool $(0.6,1.2$, and 3.6 as indicated). $P_{\mathrm{ves} 2}$ is kept constant at 0.4 . Filled symbols represent the corresponding results when there is no restriction on the number of vesicles that can be released per stimulus. The dashed line indicates $P_{\text {2rel }} / P_{2 \text { fail }}=1$.

stimulus, or not at all (Stevens and Wang, 1995), we have adopted this notion in the simulations below.

In Figure $5 A$, the relationship between $P_{1}$ and the $P_{2 \text { rel }} / P_{2 \text { fail }}$ ratio obtained by varying $P_{\text {ves } 1}$ from 0.1 to 0.9 is plotted, but only for a single value of preprimed pool (1.2) (open circles). Also included in this graph are two different kinds of simulations that take activation failures into account. In one of these, $P_{\text {ves } 1}$ is given a value of 1.0, and all variation in $P_{1}$ is thus given by activation failure (filled squares). This shift to "release failure decided by activation failure only" produces a downward shift of the curve to a value of $\sim 0.4$ (decided by the value of $P_{\text {ves } 2}$ ). In the other simulation, $P_{\text {ves } 1}$ varied in our standard manner (0.1-0.9), but in every run, $50 \%$ of the trials were (stochastically) associated with an activation failure in response to the first stimulus (filled circles). This simulation produced a curve with a ratio that is negatively correlated with $P_{1}$. However, in absolute ratio values, it is rather close to the control. It is notable that at small $P_{1}$ values, activation failures actually make the ratio closer to unity.

As elaborated in Materials and Methods, the above simulations were performed with the constraint that at most one vesicle could be released per stimulus (Triller and Korn, 1982; Korn et al., 1994; Stevens and Wang, 1995; Dobrunz and Stevens, 1997; Liu et al., 1999; Matveev and Wang, 2000; Hanse and Gustafsson, 2001b). Because this one-vesicle hypothesis is debated (Auger and Marty, 2000; Wadiche and Jahr, 2001), we also modeled the $P_{2 \text { rel }} / P_{2 \text { fail }}$ ratio when no constraints against multivesicular release were present. Figure $5 B$ shows that the release dependence of $P_{2}$ is now quite different (filled symbols), with the $P_{2 \text { rel }} / P_{2 \text { fail }}$ ratio being well below 1, and displays (for a given pool size) no relationship to $P_{1}$.

\section{Experimentally observed release dependence of $\boldsymbol{P}_{\mathbf{2}}$}

It was reported previously that $P_{2}$ was, on average among the synapses, independent of whether or not release occurred in response to the first stimulus (Stevens and Wang, 1995; Isaac et al., 1996; Hjelmstad et al., 1997; Hanse and Gustafsson, 2001b). The present simulations show that this result is compatible with synapses having small preprimed pools. They also show that in our standard release model, $P_{2}$ should depend on release to the first stimulus when $P_{\text {ves1 }}$ is small or large, with this fact creating an overall relationship between the $P_{2 \text { rel }} / P_{2 \text { fail }}$ ratio and $P_{1}$. In Figure $6 A$, the $P_{2 \text { rel }} / P_{2 \text { fail }}$ ratios obtained experimentally are plotted against the $P_{1}$ values for those synapses (open circles), demonstrating a significant $(p<0.01)$ overall relationship between the $P_{\text {2rel }} / P_{2 \text { fail }}$ ratio and $P_{1}$. Compared with the simulated values (filled circles), the experimental ones show appreciably more scatter and a smaller slope (1.13 vs 1.80). However, this larger scatter would be expected, considering that these data were obtained from runs of $\sim 100$ trials per synapse. As exemplified from one such run in Figure $6 B$ (filled circles), simulations using only 100 trials per run revealed a degree of scatter similar to that observed experimentally (open circles). The slope from a number of such runs $(100)$ averaged $1.71 \pm 0.62$ (mean $\pm \mathrm{SD})$. That is, the experimentally observed slope (1.13) is within $1 \mathrm{SD}$ of the simulated distribution. The simulated $P_{2 \text { rel }} / P_{2 \text { fail }}$ ratio, when averaged for all simulated values in Figure $6 B$, was 1.06 (i.e., showing the same overall release independence as observed experimentally).

Simulations using only 100 trials per run were also performed under conditions of variable calcium influx (Fig. $4 A$ ) and multivesicular release (Fig. $5 B$ ). In the former case, the slope of the $P_{2 \text { rel }} / P_{2 \text { fail }}$ ratio against $P_{1}$ averaged $3.13 \pm 1.32$, and the mean $P_{2 \text { rel }} / P_{2 \text { fail }}$ ratio was $1.63 \pm 0.17$. In the case of multivesicular release, the slope of the $P_{2 \text { rel }} / P_{2 \text { fail }}$ ratio against $P_{1}$ averaged $0.43 \pm 0.44$, and the mean $P_{2 \text { rel }} / P_{2 \text { fail }}$ ratio was $0.75 \pm 0.07$. Thus, in both cases, the experimentally observed mean ratio of 0.97 ( $n=33$ ) falls outside 2 SD of these distributions. Conversely, the experimentally observed slope of 1.13 is within $2 \mathrm{SD}$ of both slope distributions.

\section{DISCUSSION}

It is essential to know whether the failure of a synapse to release is attributable to the stochastic nature of the release process itself or to failure to activate the axon/synaptic bouton. A test for activation failure has been to examine the dependence of release probability on the release to a preceding stimulus, with the observation of such dependence signifying activation failure. 

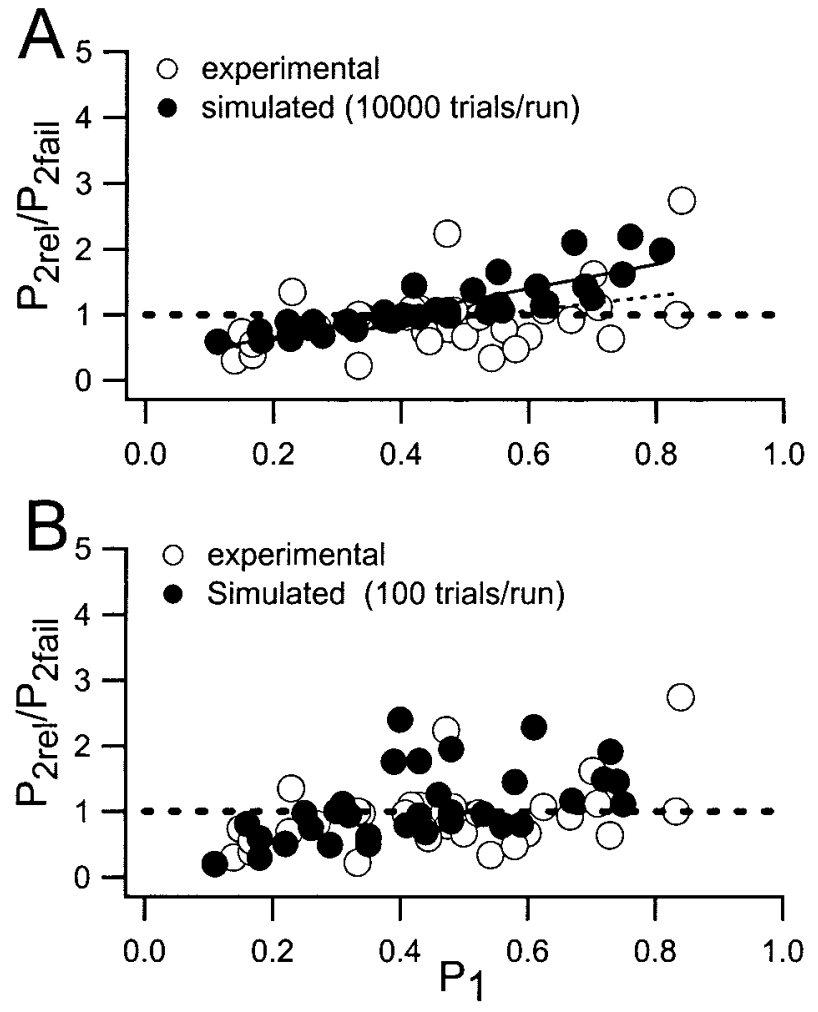

Figure 6. Comparison between experimentally observed and simulated release dependence during paired-pulse activation. $A$, The $P_{2 \text { rel }} / P_{2 \text { fail }}$ ratio is plotted against $P_{1}$ for experimental (open circles) and simulated (filled circles) data. Because the experimental data do not include synapses exhibiting the smallest and largest $P_{1}$ values, simulations were performed over a slightly smaller range of $P_{\text {ves } 1}$ values than used in the other simulations. The exclusion of those synapses depended on the fact that under conditions of very small and large $P_{1}$ values and with a limited number of trials, the number of release successes and failures, respectively, becomes too small to produce reliable results. $P_{\text {ves } 1}$ was varied between 0.2 and 0.8 (in steps of 0.1 ), the average preprimed pool was varied between 0.6 and 1.8 (in steps of 0.3 ), and $P_{\text {ves } 2}$ was kept constant at 0.4 (10,000 trials per measurement). The dashed line indicates release independence (i.e., a $P_{2 \text { rel }} / P_{2 \text { fail }}$ ratio equal to 1 ). A linear regression line is shown both for the experimental (dotted line; $y=0.39+1.13 x ; n=33$; $r=0.43 ; t_{n-2}=2.65 ; p<0.01$ ) and simulated (solid line; $y=0.29+1.85 x$; $n=35$ ) data. $B$, Same as $A$, but the simulated data were generated with a more experimentally realistic number of trials (100).

However, because of vesicle depletion produced by a preceding release, release dependence would also be expected to appear in the absence of activation failure (Kraushaar and Jonas, 2000). The present simulations demonstrate that release dependence does not need to occur even if the vesicle pool is very small. The previously observed overall absence of release dependence at hippocampal glutamatergic synapses (Stevens and Wang, 1995; Isaac et al., 1996; Hjelmstad et al., 1997; Hanse and Gustafsson, $2001 b$ ) is thus compatible with a small pool of immediately releasable vesicles (Hanse and Gustafsson, 2001c). However, the present simulations also demonstrate that for the individual synapse, release dependence is not necessarily an indicator of activation failure. Moreover, the simulations indicate that an examination of the release dependence in principle can be useful in discriminating between various models for release site functioning.

When release dependence of $P_{2}$ was experimentally studied in the neonatal rat, there was no such dependence when averaged over all the examined synapses (Hanse and Gustafsson, 2001b).
Conversely, the preprimed pool of vesicles was estimated to average only approximately one vesicle (Hanse and Gustafsson, 2001c). If such a small pool would be constant between trials, $P_{2}$ would be very release dependent, being zero whenever release had occurred in response to the first stimulus. However, in agreement with our analysis of experimental data (Hanse and Gustafsson, 2001d), the present simulations were performed using a binomially distributed trial-to-trial variation of the preprimed pool. Under these conditions, our simulations indicated little release dependence of $P_{2}$ over a range of $P_{1}$ values. This is because in a binomially distributed pool, release in response to the first stimulus will preferentially occur in those trials in which more vesicles are primed, and vice versa. The number of primed vesicles remaining for the second stimulus can then be equal independently of whether or not release occurred in response to the first stimulus.

Nevertheless, the simulations showed that a small pool leads to a release dependence when $P_{1}$ is either small or large, but in opposite directions. For a population of synapses that covers the entire range of $P_{1}$ values, these opposing effects of smaller and larger $P_{1}$ values could then approximately cancel each other out, leading to an overall release independence of $P_{2}$. Nevertheless, these simulations suggest that an experimentally observed release dependence of $P_{2}$ for an individual synapse does not by itself implicate activation failure. A larger value of $P_{1}$ was associated with a much larger $P_{2}$ when release occurred in response to the first stimulus than when it did not. Our simulations indicated that the $P_{\text {ves } 1}$ variation and the trial-to-trial variation in the preprimed pool explain this dependence on $P_{1}$. When $P_{1}$ is large, and thus $P_{\text {ves1 }}$ is large, it is very probable that release occurs whenever the preprimed pool is greater than zero. This implies that it is very probable that release failure is associated with an absence of preprimed vesicles and thus also with release failure in response to the second stimulus. Conversely, a small $P_{1}$ is associated with a small $P_{\text {ves } 1}$. Release failure then depends more on the small $P_{\text {ves } 1}$ than on the size of the preprimed pool, and vesicle depletion will more critically affect $P_{2}$.

The simulations discussed above were based on the singlevesicle constraint (see Materials and Methods) and on the as-

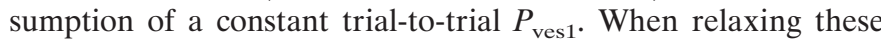
constraints, the simulations demonstrated a substantial release dependence, different in character from that discussed above. Thus, when allowing multivesicular release, there was an overall release dependence such that the average $P_{2 \text { rel }} / P_{2 \text { fail }}$ ratio was substantially below unity $(0.75)$, and there was little dependence on $P_{1}$. Conversely, the simulations using a variable (trial-to-trial) calcium influx produced an overall release dependence in the other direction.

In agreement with experimental data (Stevens and Wang, 1995; Isaac et al., 1996; Hjelmstad et al., 1997; Hanse and Gustafsson, 2001b), the simulations made using our standard release model displayed little release dependence when averaged over the entire range of $P_{1}$ values. This is in contrast to the simulations in which activation failure or multivesicular release was allowed or in which release probability was influenced by trial-to-trial fluctuations in $\mathrm{Ca}^{2+}$ influx. The experimental data thus suggest that such factors do not contribute to any large extent to the observed release of these synapses.

Both the experimental data and the simulated data displayed $P_{2 \text { rel }} / P_{2 \text { fail }}$ ratios that varied positively with $P_{1}$. The experimentally observed slope was also well within the simulated distribution of slopes. Simulations using a large pool (3.6 on average) 
indicated that such pools would not introduce any release dependence except at extreme values of $P_{1}$. As noted above, a preprimed pool that is constant for every trial also would not produce a ratio that varies with $P_{1}$. Moreover, release may be such that at most one vesicle is ever in a releasable position (Scheuss and Neher, 2001). However, $P_{2}$ would not produce a ratio that varies with the value of $P_{1}$ in this case either, because a release in response to the second stimulus would always be independent of that to the first stimulus. It would thus appear that the experimental data are not consistent with such release models.

Another feature of our release model is the assumption of equal $P_{\text {ves }}$ values for all vesicles within a release site. It has been suggested, for example, as a possible explanation for strong initial depression of release (Matveev and Wang, 2000), that one of the vesicles may have a much greater $P_{\text {ves1 }}$ than the others. The present simulations indicated that even if this $P_{\text {ves }}$ heterogeneity were substantial, such vesicle heterogeneity would not be easily detected in experimental data. However, the range of $P_{1}$ values obtained with such heterogeneity appears to become more restricted.

Chen et al. (1996) reported $P_{2 \text { rel }} / P_{2 \text { fail }}$ ratios of $>1$ and that paired-pulse facilitation was observed only when the first stimulus produced release. This was explained by assuming a large trialto-trial variation in $\mathrm{Ca}^{2+}$ influx and that only $\mathrm{Ca}^{2+}$ influx sufficient to produce release could produce residual $\mathrm{Ca}^{2+}$ levels great enough to initiate facilitation. In the present simulations, a trialto-trial variation in $\mathrm{Ca}^{2+}$ influx indeed shifted $P_{2 \text { rel }} / P_{2 \text { fail }}$ ratios to $>1$. Nevertheless, our analysis also suggests an alternative explanation. In the study by Chen et al. (1996), $P_{2 \text { fail }}$ was similar to $P_{1}$, implying that "facilitation" in fact also occurred when there was failure to the first stimulus. This is because failure trials should be biased toward smaller preprimed pools. Therefore, $P_{2 \text { fail }}$ should be smaller than $P_{1}$ unless $P_{\text {ves } 2}$ is higher than $P_{\text {ves } 1}$. A $P_{2 \text { rel }} / P_{2 \text { fail }}$ ratio of $>1$ could also be a consequence of the trial-to-trial variation in the preprimed pool at a larger $P_{1}$, as discussed above. Dobrunz et al. (1997) also reported $P_{2 \text { rel }} / P_{2 \text { fail }}$ ratios of $>1$. In contrast to the present simulations and experimental data, these ratios of $>1$ were preferentially found in low $P_{\mathrm{r}}$ synapses. A trial-to-trial fluctuation in $\mathrm{Ca}^{2+}$ influx was also favored as an explanation for these results. However, this explanation would seem to rely on differences in $P_{\text {ves1 }}$ among the synapses as underlying the heterogeneity in $P_{1}$, rather than differences in the vesicle pool size, as proposed by Dobrunz and Stevens (1997). For example, with only a small vesicle pool underlying a low $P_{1}$ value, the effect of depletion on $P_{2}$ would likely outweigh the effect of higher residual $\mathrm{Ca}^{2+}$.

In conclusion, the present simulations indicate that the releasedependence test is not useful as a test for activation failure. First, a large number of trials seem to be necessary to obtain a value for release dependence of $P_{2}$ for an individual synapse that by chance is not overlapping with that produced by some degree of activation failure. Second, release dependence can be produced by a number of other factors. Nevertheless, as indicated by the present analysis, this test may be quite useful for other purposes when data from a number of individually examined synapses are combined. Previous work has indicated that a release model based on a small, binomially distributed (trial-to-trial) pool of immediately releasable and independently acting vesicles can explain release probability (Hanse and Gustafsson, 2001c) and paired-pulse (Hanse and Gustafsson, 2001d) behavior of neonatal CA3-CA1 synapses. Without invoking additional complexity in this model, the present simulations show that the model also accounts for release dependence within a paired stimulus. It would thus appear that a rather low level of complexity is needed to explain much of the release behavior of the neonatal CA1 synapses.

\section{REFERENCES}

Allen C, Stevens CF (1994) An evaluation of causes for unreliability of synaptic transmission. Proc Natl Acad Sci USA 91:10380-10383.

Auger C, Marty A (2000) Quantal currents at single-site central synapses. J Physiol (Lond) 526:3-11.

Barbour B (2001) An evaluation of synapse independence. J Neurosci 21:7969-7984.

Chen Y, Chad JE, Wheal HV (1996) Synaptic release rather than failure in the conditioning pulse results in paired-pulse facilitation during minimal synaptic stimulation in the rat hippocampal CA1 neurones. Neurosci Lett 218:204-208.

Dobrunz LE, Stevens CF (1997) Heterogeneity of release probability, facilitation, and depletion at central synapses. Neuron 18:995-1008.

Dobrunz LE, Huang EP, Stevens CF (1997) Very short-term plasticity in hippocampal synapses. Proc Natl Acad Sci USA 94:14843-14847.

Hanse E, Gustafsson B (2001a) Factors explaining heterogeneity in short-term synaptic dynamics of hippocampal glutamatergic synapses in the neonatal rat. J Physiol (Lond) 537:141-149.

Hanse E, Gustafsson B (2001b) Quantal variability at glutamatergic synapses in area CA1 of the rat neonatal hippocampus. J Physiol (Lond) 531:467-480.

Hanse E, Gustafsson B (2001c) Vesicle release probability and preprimed pool at glutamatergic synapses in area CA1 of the rat neonatal hippocampus. J Physiol (Lond) 531:481-493.

Hanse E, Gustafsson B (2001d) Paired-pulse plasticity at the single release site level: an experimental and computational study. J Neurosci 21:8362-8369.

Hjelmstad GO, Nicoll RA, Malenka RC (1997) Synaptic refractory period provides a measure of probability of release in the hippocampus. Neuron 19:1309-1318

Isaac JT, Hjelmstad GO, Nicoll RA, Malenka RC (1996) Long-term potentiation at single fiber inputs to hippocampal CA1 pyramidal cells. Proc Natl Acad Sci USA 93:8710-8715.

Konnerth A, Keller BU, Lev-Tov A (1990) Patch clamp analysis of excitatory synapses in mammalian spinal cord slices. Pflügers Arch 417:285-290.

Korn H, Sur C, Charpier S, Legendre P, Faber DS (1994) The onevesicle hypothesis and multivesicular release. Adv Second Messenger Phosphoprotein Res 29:301-322.

Kraushaar U, Jonas P (2000) Efficacy and stability of quantal GABA release at a hippocampal interneuron-principal neuron synapse. J Neurosci 20:5594-5607.

Liu G, Choi S, Tsien RW (1999) Variability of neurotransmitter concentration and nonsaturation of postsynaptic AMPA receptors at synapses in hippocampal cultures and slices. Neuron 22:395-409.

Matveev V, Wang XJ (2000) Implications of all-or-none synaptic transmission and short-term depression beyond vesicle depletion: a computational study. J Neurosci 20:1575-1588.

McAllister AK, Stevens CF (2000) Nonsaturation of AMPA and NMDA receptors at hippocampal synapses. Proc Natl Acad Sci USA 97:6173-6178.

Raastad M, Storm JF, Andersen P (1992) Putative single quantum and single fibre excitatory postsynaptic currents show similar range and variability in rat hippocampal slices. Eur J Neurosci 4:113-117.

Sakaba T, Neher E (2001) Quantitative relationship between transmitter release and calcium current at the calyx of held synapse. J Neurosci 21:462-476.

Scheuss V, Neher E (2001) Estimating synaptic parameters from mean, variance, and covariance in trains of synaptic responses. Biophys $\mathbf{J}$ 81:1970-1989.

Stevens CF, Wang Y (1995) Facilitation and depression at single central synapses. Neuron 14:795-802.

Triller A, Korn H (1982) Transmission at a central inhibitory synapse. III. Ultrastructure of physiologically identified and stained terminals. J Neurophysiol 48:708-736.

Wadiche JI, Jahr CE (2001) Multivesicular release at climbing fiberPurkinje cell synapses. Neuron 32:301-313. 